\title{
LGR4: A new receptor for a stronger bone
}

\author{
Yunyun Jin \& Yingzi Yang \\ Department of Developmental Biology, Harvard School of Dental Medicine, Boston, MA 02115, USA
}

Received April 16, 2016; accepted April 26, 2016; published online May 12, 2016

Citation: Jin, Y., and Yang, Y. (2016). LGR4: A new receptor for a stronger bone. Sci China Life Sci 59, 735-736. doi: 10.1007/s11427-016-5068-8

Bone formation and remodeling involves production of bone matrix by osteoblasts and its resorption by osteoclasts. Increased osteoclast activity or reduced osteoblast function leads to osteopenic disorders (Kong et al., 1999). Therefore, it is crucial to understand mechanisms underlying osteoclast regulation. Such understanding will shed light on identifying potential therapeutic targets of osteoporosis, a disease caused by too much bone resorption or insufficient bone formation. RANKL-RANK signaling is required for osteoclast, activating a variety of downstream signaling pathways. RANK, also known as tumor necrosis factor (TNF) superfamily member 11 (TNFSF11), has long been considered to be the sole receptor for RANKL, also known as TNFRSF11A, (Hanada et al., 2011). In a recent study, Mingyao Liu and Jian Luo, Jianru Xiao, et al., at East China Normal University, have discovered that the leucine-rich repeat-containing G-protein-coupled receptor 4 (LGR4, also called GPR48) is a novel receptor for RANKL, in that it competes with RANK for RANKL binding in osteoclasts, and that it negatively regulates osteoclast differentiation and bone remodeling (Luo et al., 2016). This research, published in Nature Medicine, provides the first evidence that a RANKL-LGR4-G $\alpha_{\mathrm{q}}$-GSK3- $\beta$-NFATC1 signaling pathway regulates osteoclastogenesis and suggests that LGR4 is a candidate for treating osteoporosis and other boneresorption diseases. This finding provides a molecular mechanism explanation to the low bone density of LGR4deficiency in humans and mice, as well as to many other potential defects caused by LGR4 mutation.

The $\operatorname{Tnf} f f 11^{-/-}$mice exhibit the osteopetrosis phenotype

email: Yunyun_Jin@hsdm.harvard.edu caused by reduced bone resorption, and it also showed disrupted immune regulation, and failure of mammary gland lobuloalveolar development. Liu's team noticed that the $\mathrm{LrH}^{-/-}$mice shared some similar sets of phenotypes with the Tnfsf $11^{-/-}$mice, thus suggesting that LGR4 lies in the same pathway as RANKL-RANK (Luo et al., 2016). This team then demonstrated, with rigorous biochemical assays, that RANKL directly binds human LGR4, but that RANK doesn't bind LGR4. The team further showed that the extracellular domain (ECD) of LGR4 mediates the specific interaction between LGR4 and RANKL.

G-protein-coupled receptors (GPCRs or GPRs) are integral membrane proteins involved in the transmission of signals from the extracellular environment to the cytoplasm. GPCRs are important drug targets. Predicted to be a G protein-coupled receptor (GPCR), LGR4 is also associated with Wnt receptors and it mediates $\mathrm{R}$-spondin signaling. R-spondins (RSPOs) have been reported as functional ligands for LGR4 in adult stem cell maintenance and activity. RSPO binding to LGR4 only activates Wnt signaling. Therefore, additional LGR4 ligands are responsible for the activation of G-protein signaling pathways downstream of LGR4 which are implicated in eye development, innate immunity, energy expenditure, and osteoblast differentiation. This is so, even though previous studies showed that the ligands of LGR4 failed to activate heterotrimeric G-proteins to transduce the signal (Carmon et al., 2011). Interestingly, the authors found that LGR4 activates $G \alpha_{q}$ when binding with RANKL and induces intracellular calcium release. This is the first report showing G-protein signaling can be induced via ligand activated LGR4.

Similar to RANKL, LGR4 is shown to regulate osteo- 
clast differentiation. By analyzing bone phenotypes of two mutant mice lines-whole-body $\left(\mathrm{Lgr}^{-/-}\right)$and monocyte conditional mutant mice of Lgr4 (Lgr4 CKO)- the authors found that hyperactivation of osteoclasts, as shown by greater numbers of TRAP-positive osteoclasts and larger osteoclast size in the femoral bones and calvaria bones in vivo. Further more, the authors also provided in vitro evidences that loss of Lgr4 in the bone marrow monocytes (BMMs) from Lgr4 CKO mice enhances the formation of osteoclast and accelerates BMMs differentiation when treated with RANKL. In addition, LGR4 also regulates osteoclast survival by inducing apoptosis. The authors' finding mechanically explained the phenomenon that the mature osteoclasts finally undergo apoptosis even in an environment with RANKL.

In osteoblasts, Lgr4 appears to have a different function. Liu's team showed that Lgr4 acts through the cAMP-PKACREB pathway to regulate the expression level of Atf4 in osteoblasts, showing that Lgr4 is involved in the osteoblast differentiation and bone formation (Luo et al., 2009). Compared with its function in the osteoclastogenesis, they do suggest that the osteoclast deficiency is the major cause of low bone mass seen in mice and humans with an LGR4 mutation.

RSPOs and Norrin are the ligands of LGR4. The authors found that RSPOs and Norrin are not involved in Lgr4deficiency-induced osteoclastogenesis. Instead, LGR4 regulates canonical RANKL-RANK signaling through: (i) LGR4-ECD competes with RANK to bind with RANKL, (ii) LGR4 decreases interaction between RANK and a downstream component TRAF6, (iii) LGR4 abrogated RANKL- induced NF- $\kappa B$ signaling.

The transcription factor NFATC1 is a master regulator of RANKL-induced osteoclastogenesis, when NFATC1 is expressed in precursor cells. By overexpression of a constitutively active form of $\mathrm{G} \alpha_{\mathrm{q}}\left(\mathrm{G} \alpha_{\mathrm{q}}{ }^{\mathrm{CA}}\right)$, the authors showed that $\mathrm{G}_{\mathrm{q}}{ }^{\mathrm{CA}}$ blocked Lgr4-knock-down-induced osteoclast differentiation via decreasing phosphorylation of glycogen synthase kinase $3-\beta$ (GSK3- $\beta$ ). GSK3- $\beta$ phosphorylation inhibits GSK3- $\beta$ activity that promotes NFATC1nuclear localization. These observations led to the identification of a new RANKL-LGR4-G $\alpha_{\mathrm{q}}$-GSK3- $\beta$-NFATC1 pathway in osteoclastogenesis. They also demonstrated that LGR4 expression is induced by RANKL-NFATC1 signaling during osteoclast differentiation, thus indicating a negative-feedback mechanism exists to limit osteoclastogenesis.

The soluble LGR4-ECD protein might be used to treat osteoporosis as it can block osteoclast differentiation induced by RANKL. The authors showed that in ovariectomy, RANKL injected and Tnfrsfllb-deficient osteoporosis mouse models, treated with LGR4-ECD, increased bone mass, but LGR4-ECD protein had little effect on osteoclast differentiation in wild type mice. A mechanistic understanding of RANKl-LGR4 interaction has provided new insight into LGR4 mediated RANKL signaling in osteoporosis and other diseases.

Compliance and ethics The author(s) declare that they have no conflict of interest.

Carmon, K.S., Gong, X., Lin, Q., Thomas, A., and Liu, Q. (2011). R-spondins function as ligands of the orphan receptors LGR4 and LGR5 to regulate Wnt/beta-catenin signaling. Proc Natl Acad Sci USA $108,11452-11457$.

Hanada, R., Hanada, T., Sigl, V., Schramek, D., and Penninger, J.M. (2011). RANKL/RANK-beyond bones. J Mol Med (Berl). 89, 647-656.

Kong, Y.Y., Yoshida, H., Sarosi, I., Tan, H.L., Timms, E., Capparelli, C., Morony, S., Oliveira-dos-Santos, A.J., Van, G., Itie, A., Khoo, W., Wakeham, A., Dunstan, C.R., Lacey, D.L., Mak, T.W., Boyle, W.J., and Penninger, J.M. (1999). OPGL is a key regulator of osteoclastogenesis, lymphocyte development and lymph-node organogenesis. Nature 397, 315-323.

Luo, J., Yang, Z., Ma, Y., Yue, Z., Lin, H., Qu, G., Huang, J., Dai, W., Li, C., Zheng, C., Xu, L., Chen, H., Wang, J., Li, D., Siwko, S., Penninger, J.M., Ning, G., Xiao, J., and Liu, M. (2016). LGR4 is a receptor for RANKL and negatively regulates osteoclast differentiation and bone resorption. Nat Med doi: 10.1038/nm.4076.

Luo, J., Zhou, W., Zhou, X., Li, D., Weng, J., Yi, Z., Cho, S. G., Li, C., Yi, T., Wu, X., Li X.Y., de Crombrugghe B., Hook M., and Liu, M. (2009). Regulation of bone formation and remodeling by G-protein-coupled receptor 48. Development 136, 2747-2756

Open Access This article is distributed under the terms of the Creative Commons Attribution License which permits any use, distribution, and reproduction in any medium, provided the original author(s) and source are credited. 